\title{
microRNA-155 promotes the proliferation of prostate cancer cells by targeting annexin 7
}

\author{
ZHI-KANG CAI ${ }^{*}$, QI CHEN*, YAN-BO CHEN, MENG GU, DA-CHAO ZHENG, JUAN ZHOU and ZHONG WANG \\ Department of Urology and Andrology, Ninth People's Hospital, School of Medicine, \\ Shanghai Jiaotong University, Shanghai 200011, P.R. China
}

Received November 25, 2013; Accepted June 26, 2014

DOI: $10.3892 / \mathrm{mmr} .2014 .2744$

\begin{abstract}
Micro (mi)RNAs are a group of small non-coding RNA molecules that have been demonstrated to regulate the expression of genes involved in tumorigenesis. The relevance of microRNAs in the development, progression and prognosis of prostate cancer is not fully understood. miR-155 has been implicated in the induction of breast, lung and liver cancer, but its role in prostate cancer has not been investigated. In the present study, the biological function of miR-155 was investigated in prostate cancer for the first time, to the best of our knowledge. It was demonstrated that the expression of miR-155 was upregulated in prostate cancer tissues and cell lines as determined by quantitative reverse transcription-polymerase chain reaction. Furthermore, overexpression of miR-155 promoted cell proliferation, as indicated by MTT assay. Flow cytometric analysis demonstrated that inhibition of miR-155 induced cell cycle arrest and promoted apoptosis in prostate cancer cells. In addition, western blot analysis indicated that annexin (ANX)7 was significantly downregulated in prostate cancer tissues and cells. A luciferase reporter assay indicated that ANX7 was a target of miR-155, which suggested that miRNA-155 promoted the proliferation of prostate cancer cells by regulating ANX7 expression levels.
\end{abstract}

\section{Introduction}

Prostate cancer is one of the most common non-skin malignancies in males, and the morbidity and mortality are increasing significantly in China. However, the incidence of prostate cancer in China remains low compared with incidences in Western countries, including the USA and those in Europe $(1,2)$. Currently,

Correspondence to: Dr Zhong Wang, Department of Urology and Andrology, Ninth People's Hospital, School of Medicine, Shanghai Jiaotong University, 639 Zhi Zaoju Road, Shanghai 200011, P.R. China

E-mail: zhongwang2010@sina.com

*Contributed equally

Key words: prostate cancer, cell proliferation, miR-155, annexin 7 digital rectal examination, prostate-specific antigen testing and histopathological evaluation of prostate needle biopsies are all used to detect and monitor prostate cancer progression $(3,4)$. The loss of normal glandular tissue is measured by the prostate cancer scoring system called the Gleason score, which aims to assist the evaluation of patient prognosis (5). However, prostate cancer progression is variable and occasional cases behave independently of their Gleason scores (6). Therefore, further studies of the molecular pathogenesis of prostate cancer are required in order to develop novel and effective biomarkers to improve the prediction of cancer prognoses.

microRNAs (miRNAs) are a class of small $(<22 \mathrm{nt})$, non-coding RNA molecules that are essential in numerous biological processes (7). Accumulating evidence has demonstrated that miRNAs have the ability to regulate the expression of a variety of genes involved in tumorigenesis and are critical in the processes of cell proliferation, differentiation and apoptosis $(8,9)$. In animals, miRNAs have been indicated to produce regulatory effects through binding to the complementary sequences within the 3'-untranslated regions (3'UTRs) of their mRNA targets. A previous study suggested that miRNAs may be responsible for the regulation of more than one-third of all human genes (10). miR-155 is a particularly important miRNA. It is produced from the processing of the B-cell integration cluster, has been observed to be overexpressed in numerous types of cancer, and is linked to the development of leukemia, breast and lung cancer $(11,12)$. However, the biological function and specific mechanism of action of miR-155 in prostate cancer has yet to be elucidated. Therefore, in the present study, the clinical relevance of miR-155 in prostate cancer was examined.

\section{Materials and methods}

Cell culture and tissue samples. The PNT1B human prostate normal tissue cell line and human prostate cancer cell lines DU145, LNCaP, 22RV1 and PC-3 were obtained from the Chinese Academy of Sciences (Shanghai, China). All cell lines used were cultured in RPMI 1640 medium (Gibco Life Technologies, Beijing, China) supplemented with $10 \%$ fetal calf serum, $100 \mathrm{IU} / \mathrm{ml}$ penicillin and $100 \mathrm{mg} / \mathrm{ml}$ streptomycin (Gibco Life Technologies). A total of 35 paired human prostate cancer and adjacent non-tumor tissues were collected from routine therapeutic surgery at our department. Written informed consent was obtained from all participants 
involved in the present study. The study was performed in accordance with the Declaration of Helsinki and approved by the Institutional Review Board of the Ninth People's Hospital, School of Medicine, Shanghai Jiaotong University (Shanghai, China).

RNA extraction and quantitative polymerase chain reaction $(q P C R)$. Cells were seeded onto 12-well plates and total RNA was isolated from tissues or cells with TRIzol reagent (Invitrogen Life Technologies, Carlsbad, CA, USA) according to the manufacturer's instructions. The quantity and concentration of RNA were determined by ultraviolet (UV) measurement of the optical density (OD) at 260/280 nm using the NanoDrop 2000 UV-Vis spectrophotometer (Thermo Fisher Scientific, Waltham, MA, USA). In order to quantify the expression of miR-155, the isolated RNA was reverse-transcribed and amplified using the Real Time-PCR miRNA Detection kit (Ambion Life Technologies, Carlsbad, MA, USA) according to the manufacturer's instructions. PCR reactions were performed with an Applied Biosystems 7500 Real-Time PCR system (Foster City, CA, USA) using the following cycling conditions: $95^{\circ} \mathrm{C}$ for $10 \mathrm{~min}$, then 40 cycles of $95^{\circ} \mathrm{C}$ for $15 \mathrm{sec}$ and $60^{\circ} \mathrm{C}$ for $1 \mathrm{~min}$. The U6 small nuclear RNA (Sigma-Aldrich, St. Louis, MO, USA) was used as a loading control. GAPDH was used as a control in the PCR reactions. The miRNA-specific primers were designed using Primer Premier 5.0 (Premier Biosoft International, Palo Alto, CA, USA), and the primer sequences were as follows: miR-155 sense, 5'-ACACTCCAGCTGGGTAGCTTATCAGACT-3' and antisense, 5'-CTCAACTGGTGTCGTGGAGTCGGCAA-3'; U6 sense, 5'-CTCGCTTCGGCAGCACA-3 and antisense, 5'-AACGCTTCACGAATTTGCGT-3'.

MTT assay. Proliferation of cells was determined by MTT assay. Briefly, the prostate cancer cell lines LNCaP and PC-3 transfected with oligonucleotides were seeded into 96 -well plates at a density of $3 \times 10^{4}$ cells/well. Next, $10 \mathrm{ml} \mathrm{MTT}(5 \mathrm{mg} / \mathrm{ml})$ was added and incubated in the dark at $37^{\circ} \mathrm{C}$ for $2 \mathrm{~h}$. The absorbance was determined at a wavelength of $492 \mathrm{~nm}$.

Apoptosis and cell cycle analysis. At $48 \mathrm{~h}$ post-transfection with the miR-155 precursor/inhibitor or control, LNCaP and PC-3 cells were washed with phosphate-buffered saline (PBS), detached with trypsin and harvested. The cells were resuspended in $1 \mathrm{ml}$ Hoechst 33258 stain (BD Biosciences, San Diego, CA, USA) for $5 \mathrm{~min}$ and then washed with PBS three times. Apoptotic cells were evaluated using the annexin V-fluorescein isothiocyanate/propidium iodide (PI) cell Apoptosis Detection kit (BD Biosciences, Franklin Lakes, NJ, USA) following the manufacturer's instructions. For cell cycle analysis, cells transfected with oligonucleotides were washed twice with PBS and collected by centrifugation, followed by fixation in ice-cold $70 \%$ ethanol at $-20^{\circ} \mathrm{C}$ overnight. Cells were collected and stained with $100 \mu \mathrm{l}$ PI staining solution for $30 \mathrm{~min}$ in the dark prior to cell cycle analysis.

Western blot. Cultured cells were lysed using radioimmunoprecipitation assay buffer (Sigma-Aldrich) and tissue samples were lysed using the T-PER Tissue Protein Extraction reagent (Pierce, Rockford, IL, USA) in the presence of Halt Protease Inhibitor Cocktail (Pierce). The tissue and cell lysates were then subjected to SDS-PAGE. For immunoblotting, the membranes were blocked with $5 \%$ non-fat milk in Tris-buffered saline (Sigma-Aldrich) and then incubated with mouse anti-human annexin (ANX)7 monoclonal antibody (Abcam, Cambridge, MA, USA) followed by horseradish peroxidase-conjugated secondary antibody (Abcam). Signals with Immobilon immunoreactive bands were detected with an Enhanced Chemiluminescence (ECL) Western Blot kit (Sigma-Aldrich) using a Chemigenius Bioimaging system (Syngene, Frederick MD, USA). GAPDH contents were measured as a loading control.

Plasmid construction and luciferase activity assay. Luciferase reporters were generated based on the firefly luciferase-expressing vector pMIR-Report (Ambion, USA). The wild-type and mutant ANX7-3'UTRs were amplified using the following primers, designed by Primer Premier 5.0: Wild-type forward, 5'-ATAGCATCGCAGGTA ACGCTACGCGGGACG-3' and reverse, 5'-GCAGGTAGC CGACATCGTACGCGATATACT-3'; mutant type forward, 5'-ATGCCGATGCTCGATCATACTACAGTAGCT-3' and reverse, 5'-GACGCCGTACTCGACAGCGTCGTA TGAGTG-3'. Cells were seeded into 24-well plates at a density of $5 \times 10^{4}$ cells/well one day prior to transfection. Luciferase reporter (500 ng), miRNA-155 precursor or negative control (NC) (50 pmol) and pRL-TK (40 ng) were added to each well. Cells were collected $48 \mathrm{~h}$ after transfection and analyzed using the Dual-Luciferase Reporter Assay system (Promega, Madison, WI, USA).

Statistical analysis. All values are presented as the mean \pm standard error and analyzed by SPSS version 13.0 (IBM, Armonk, NY, USA). For comparisons between two different groups, statistical significance was determined using Student's t-test. Comparisons among groups were performed using analysis of variance. $\mathrm{P}<0.05$ was considered to indicate a statistically significant difference.

\section{Results}

MiR-155 is upregulated in prostate cancer tissue and cell lines. To investigate the biological role of miR-155 in prostate cancer, the expression levels of miR-155 were determined in 35 paired prostate cancer and adjacent non-tumor tissues by qPCR. The results demonstrated that the expression of miR-155 was significantly upregulated in cancer tissues compared with the matched non-tumor samples (Fig. 1A). Additionally, the human prostate normal tissue cell line PNT1B and several prostate cancer cell lines, including LNCaP, DU145, PC-3 and 22RV1, were analyzed by qPCR. As shown in Fig. 1B, a significant upregulation of miR-155 was also observed in the four prostate cancer cell lines compared with the PNT1B cells. Taken together, these results suggested that miR-155 was significantly upregulated in prostate cancer tissues and cell lines.

Effects of miR-155 on prostate cancer cell proliferation. As the expression of miR-155 was markedly increased 
A

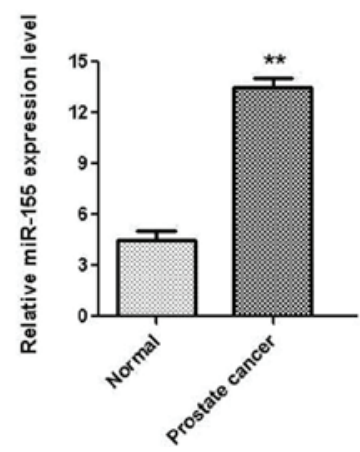

B

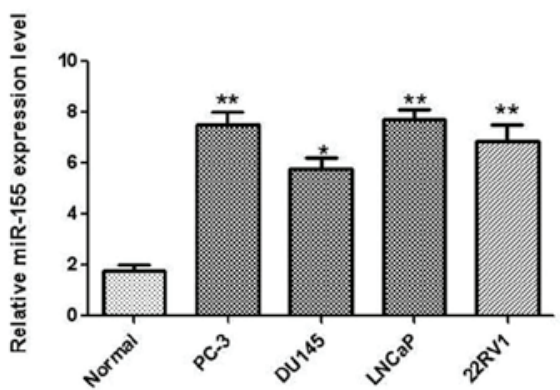

Figure 1. Upregulation of miR-155 in prostate cancer tissues and cell lines. (A) qPCR was performed to determine the miR-155 expression levels in 35 paired human prostate cancer and adjacent non-tumor tissues. ${ }^{*} \mathrm{P}<0.05 ;{ }^{* *} \mathrm{P}<0.01$. (B) The human prostate normal tissue cell line PNT1B and several prostate cancer cell lines, including LNCaP, DU145, PC-3 and 22RV1, were analyzed by qPCR to measure miR-155 expression levels. " $\mathrm{P}<0.05$; ${ }^{*}$ " $\mathrm{P}<0.01$, compared to normal. qPCR, quantitative polymerase chain reaction; miR, microRNA.

A

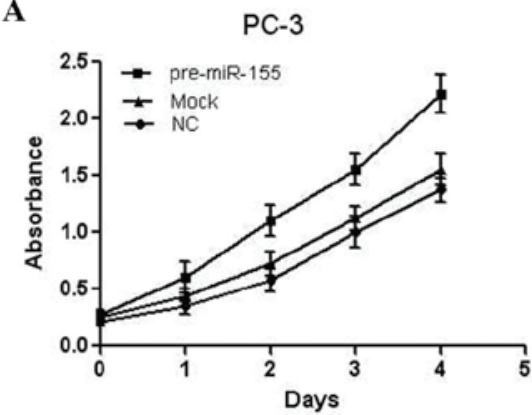

$\mathbf{C}$

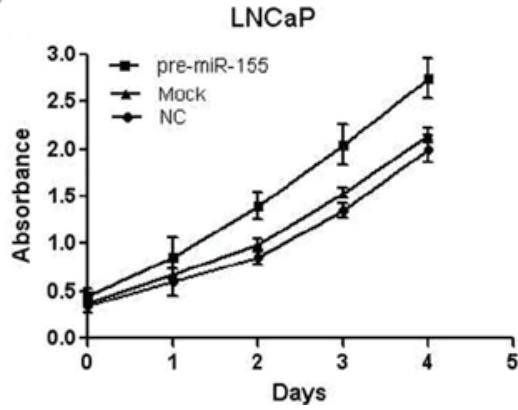

B

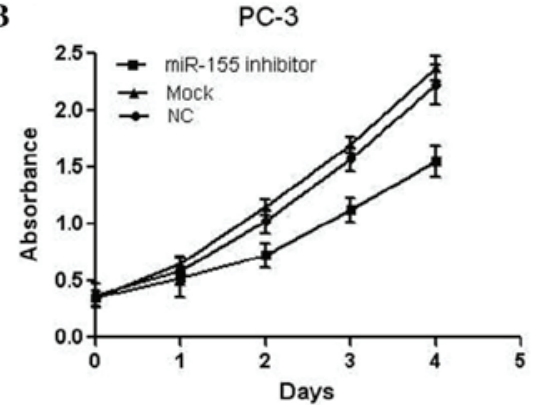

D

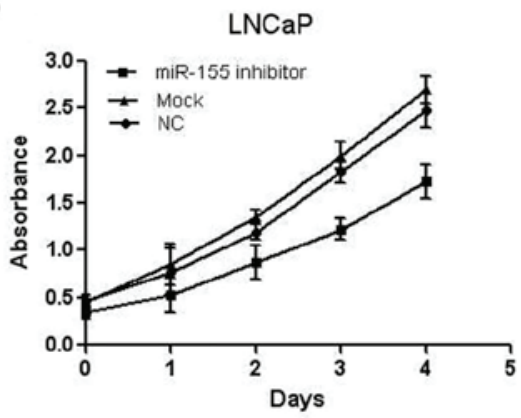

Figure 2. Effects of miR-155 on prostate cancer cell proliferation. PC-3 human prostate cancer cells were transfected with a miR-155 (A) precursor or (B) inhibitor and the MTT assay was performed every $24 \mathrm{~h}$ to measure the levels of cell proliferation. Similarly, LNCaP prostate cancer cells were transfected with a miR-155 (C) precursor or (D) inhibitor followed by MTT assay to measure the levels of cell proliferation. NC, normal control; miR, microRNA.

in prostate cancer, it was reasonable to hypothesize that it may function as a tumor promoter. Therefore, the effect of overexpression/inhibition of miR-155 on the cell proliferation of the prostate cancer cell lines was assessed. PC-3 cells were transfected with miR-155 precursor and the cell proliferation was measured by MTT assay. As presented in Fig. 2A, miR-155 overexpression significantly promoted the proliferation of PC-3 cells. PC-3 cells were also transfected with miR-155 inhibitor, and an MTT assay indicated reduced proliferation of the cells (Fig. 2B). Similar results were also observed in LNCaP cells (Fig. 2C and D). Collectively, these results demonstrated that miR-155 was able to promote the proliferation of prostate cancer cell lines in vitro.

Inhibition of miR-155 promotes apoptosis of prostate cancer cells. The effect of miR-155 on the apoptosis of human prostate cancer cells was investigated by flow cytometry. Two prostate cancer cell lines, PC-3 and LNCaP, were transfected with miR-155 inhibitor and the apoptotic rate was analyzed using Annexin V and PI staining. The results indicated that downregulation of miR-155 led to a significant increase in the apoptotic rates in PC-3 (Fig. 3A) and LNCaP (Fig. 3B) prostate cancer cells.

MiR-155 downregulation induces prostate cancer cell cycle arrest. Human prostate cancer cells were transfected with miR-155 inhibitor to investigate the effect of miR-155 on cell cycle distribution. In PC-3 cells, treatment with miR-155 inhibitor significantly increased the proportion of cells in $\mathrm{G}_{1}$ phase and reduced the number of cells in $\mathrm{S}$ phase (Fig. 4A and B). Similar results were also observed in $\mathrm{LNCaP}$ cells following transfection with the miR-155 inhibitor (Fig. 4C and D). These 
A

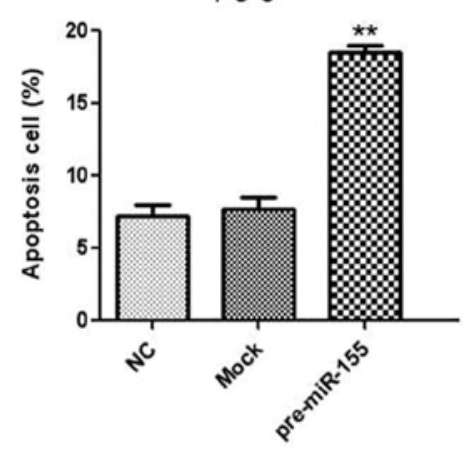

B

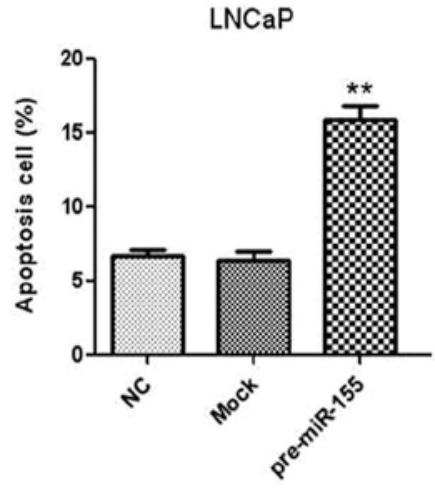

Figure 3. Inhibition of miR-155 promotes prostate cancer cell apoptosis. The effect of miR-155 on cell apoptosis of human prostate cancer cells was investigated by flow cytometry. Apoptotic rates were determined in the two prostate cancer cell lines (A) PC-3 and (B) LNCaP following transfection with miR-155 inhibitor. ${ }^{*} \mathrm{P}<0.05 ;{ }^{* *} \mathrm{P}<0.01$, compared to NC. NC, normal control; miR, microRNA.

A

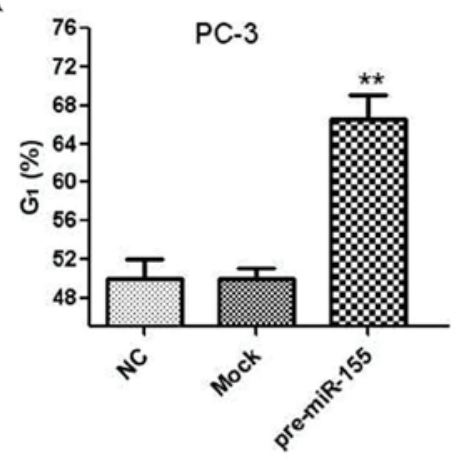

C

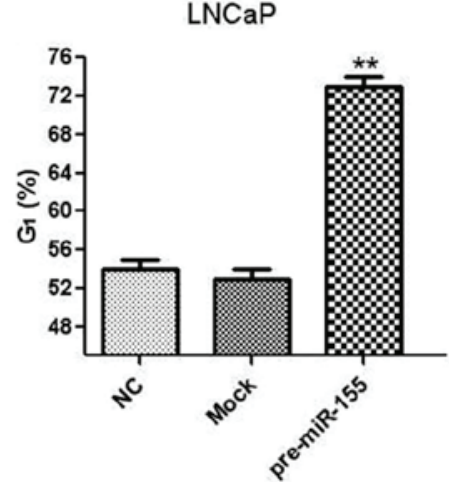

B

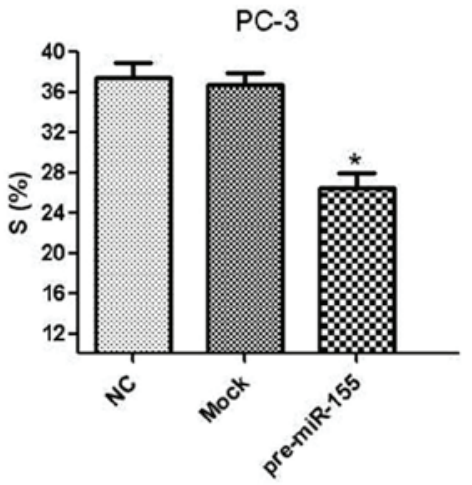

D

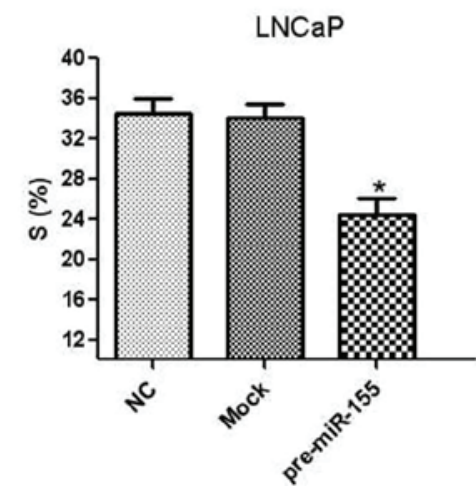

Figure 4. MiR-155 inhibition induced prostate cancer cell cycle arrest. Human prostate cancer cells were transfected with miR-155 inhibitor. The role of miR-155 on cell cycle distribution in human prostate cancer cells was determined by flow cytometry. (A) $\mathrm{G}_{1}$ and (B) $\mathrm{S}$ refer to the cycle phase of PC-3 cells (C and D) Cell cycle distribution of LNCaP cells. ${ }^{*} \mathrm{P}<0.05 ;{ }^{* *} \mathrm{P}<0.01$, compared to NC. NC, normal control; miR, microRNA.

results demonstrated that inhibition of miR-155 induced cell cycle arrest in $\mathrm{G}_{1}$ phase in human prostate cancer cells.

ANX7 is a target ofmiRNA-155 in prostate cancer cells. Toelucidate the molecular mechanism by which miRNA-155 enhanced prostate cancer cell proliferation, putative miRNA-155 targets were identified using a number of programs, including miRanda (http://www.microrna.org/microrna/home.do), TargetScan (http://www.targetscan.org/)and PicTar (http://pictar. mdc-berlin.de/). A 3'UTR of ANX7 containing the conserved putative miRNA-155 binding sites was identified. Western blot analysis indicated that the ANX7 expression levels were significantly reduced in prostate tissues (Fig. 5A) and cancer cells (Fig. 5B and C). Firefly luciferase activity was significantly increased in PC-3 cells following transfection with miR-155 inhibitor (Fig. 5D). In addition, treatment of PC-3 cells with miR-155 inhibitor led to a significant increase in the ANX7 expression (Fig. 5E and F). Together, these results suggested that the ANX7-3'-UTR is located at direct binding sites of miR-155.

\section{Discussion}

miRNAs are a group of small non-coding RNAs that modulate gene expression by targeting mRNAs for translational repression $(13,14)$. The expression of miR-155 has been demonstrated 
A

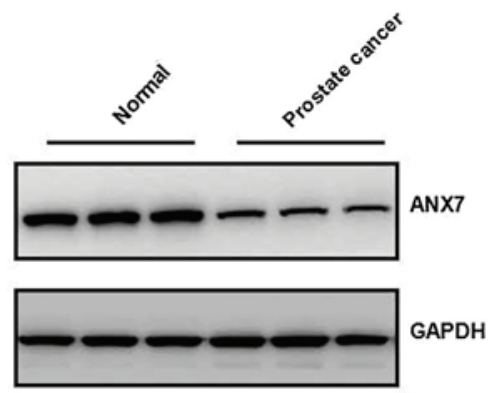

D

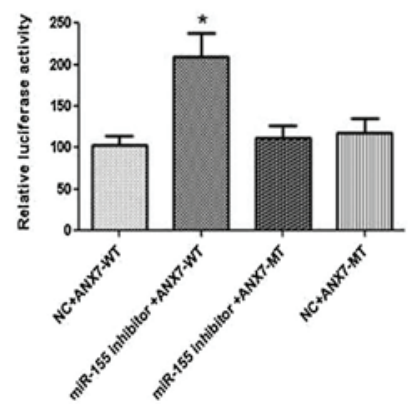

B

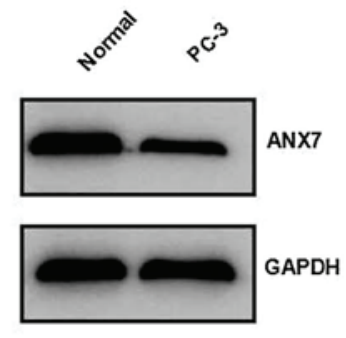

E

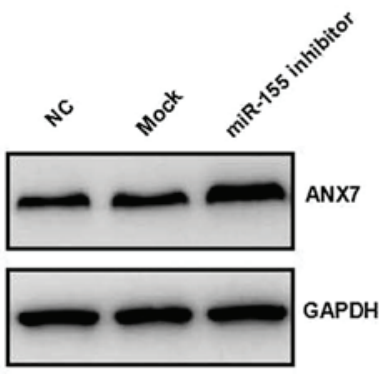

C

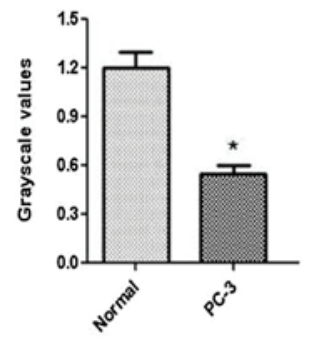

F

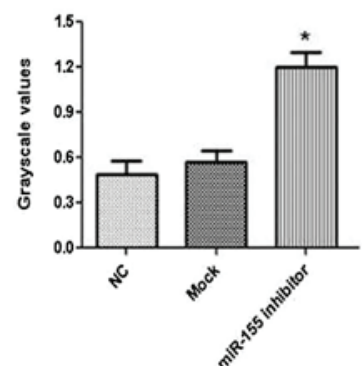

Figure 5. ANX7 is a target of miRNA-155 in prostate cancer cells. (A) Western blotting was performed to determine the ANX7 expression levels in prostate cancer and adjacent non-tumor tissues. (B and C) The protein expression of ANX7 in a normal human prostate cell line and a prostate cancer cell line were analyzed by western blot. "P<0.05, compared to normal. (D) Luciferase activity levels were determined in PC-3 cells following transfection with miR-155 inhibitor and WT/MT ANX7-3'-UTR. "P<0.05, compared to normal. (E and F) Western blot was used to measure the ANX7 expression levels in PC-3 cells following transfection with miR-155 inhibitor and WT/MT ANX7-3'-UTR. "P $<0.05$, compared to NC. ANX7, annexin 7; miR, microRNA; UTR, untranslated region; WT, wild-type; MT, mutant type.

to be upregulated in numerous types of cancer, and is involved in the development of leukemia, breast and lung cancer $(11,12)$. However, the biological role of miR-155 in prostate cancer has not been investigated, to the best of our knowledge. In the present study the expression patterns of miR-155 were investigated in prostate cancer for the first time. The results indicated that miR-155 was significantly upregulated in prostate cancer tissues and four different cell lines (DU145, LNCaP, 22RV1 and PC-3).

The aberrant expression of miRNAs is important in tumor cell proliferation, apoptosis and cell cycle arrest. A recent study indicated that miR-125b, which is overexpressed in prostate cancer tissues and cells, promoted the growth of a prostate cancer xenograft by downregulation of the key pro-apoptotic genes, including p53, p35 upregulated modulator of apoptosis and B-cell lymphoma 2 homologous antagonist killer (15). Another study reported that miR-155 promoted the proliferation of human breast cancer MCF-7 cells by targeting p53-induced nuclear protein 1 , and therefore created a novel therapeutic strategy for breast cancer (16). In the present study, an MTT assay revealed that overexpression of miR-155 significantly promoted cell proliferation of PC-3 and LNCaP cells. Additionally, human prostate cancer cells transfected with miR-155 inhibitor exhibited reduced proliferation, suggesting that miR-155 promoted prostate cancer cell proliferation in vitro.

The effect of miR-155 on levels of cell apoptosis and cell cycle distribution in prostate cancer cells was also examined. Apoptosis is the process of programmed cell death that is crucial for cell growth and the maintenance of cellular homeostasis, and is regulated by numerous signaling pathways (17). Cell cycle processes are precise, and controlled by cell cycle checkpoints which guarantee the fidelity of cell division. However, cell cycle arrest is triggered by various factors that lead to cell death and apoptosis (18). Petrocca et al (19) demonstrated that the miR-106b-25 cluster is indispensible in the development of transforming growth factor- $\beta$-dependent cell-cycle arrest and apoptosis in gastric cancer. Another study demonstrated that miR-155 downregulation induced cell apoptosis by targeting many anti-apoptotic factors, leading to cell cycle arrest (20). The present study revealed that inhibition of miR-155 promoted apoptosis of PC-3 and LNCaP prostate cancer cell lines. Transfection with an miR-155 inhibitor significantly increased the proportion of cells in $\mathrm{G}_{1}$ phase and reduced that in $\mathrm{S}$ phase, demonstrating that low expression of miR-155 induced cell cycle arrest in $\mathrm{G}_{1}$ phase.

miRNAs control a number of biological functions of the cell by targeting various genes and altering their expression levels; therefore, it is critical to elucidate the functional targeted genes. The annexin family consists of a group of proteins with a common structure that contains two distinct regions, an annexin core and an amino (N)-terminus (21). Annexins are involved in the regulation of membrane trafficking, cellular adhesion and tumorigenesis $(22,23)$. Annexin 7 (ANX7) is a substrate for protein kinase $\mathrm{C}$ and other kinases associated with cell survival, proliferation, differentiation and cell migration (24). Srivastava et al (25) have demonstrated that reduced expression of ANX7 may be associated with the progression of prostate cancer, indicating that ANX7 functions as a tumor suppressor. Consistent with previous studies, the present study observed that ANX7 expression levels were significantly reduced in prostate cancer samples and cell lines. In addition, transfection with an miR-155 inhibitor led to increased luciferase activity and ANX7 
expression in prostate cancer cells, indicating that ANX7 is a target gene of miR-155.

In conclusion, the present study demonstrated that miRNA-155 was significantly upregulated in prostate cancer tissues and cell lines. In addition, miRNA-155 was indicated to promote the proliferation of prostate cancer cells by regulating ANX7 expression levels, indicating the potential for the therapeutic use of miRNA-155 in the treatment of prostate cancer.

\section{Acknowledgements}

The present study was supported by a project of the Science and Technology Commission of Shanghai Municipality (no. 134119a9800) and the National Natural Science Foundation of China (nos. 81172450 and 81202008).

\section{References}

1. Dunn MW and Kazer MW: Prostate cancer overview. Semin Oncol Nurs 27: 241-250, 2011.

2. Ha HK, Lee W, Park HJ, et al: Clinical significance of CXCL16/CXCR6 expression in patients with prostate cancer. Mol Med Rep 4: 419-424, 2011.

3. Gosselaar C, Roobol MJ, Roemeling S, van der Kwast TH, and Schröder FH: Screening for prostate cancer at low PSA range: the impact of digital rectal examination on tumor incidence and tumor characteristics. Prostate 67: 154-161, 2007.

4. Katahira K, Takahara T, Kwee TC, et al: Ultra-high-b-value diffusion-weighted MR imaging for the detection of prostate cancer: evaluation in 201 cases with histopathological correlation. Eur Radiol 21: 188-196, 2011.

5. Stewart GD, Gray K, Pennington CJ, et al: Analysis of hypoxia-associated gene expression in prostate cancer: lysyl oxidase and glucose transporter-1 expression correlate with Gleason score. Oncol Rep 20: 1561-1567, 2008.

6. Arora R, Koch MO, Eble JN, et al: Heterogeneity of Gleason grade in multifocal adenocarcinoma of the prostate. Cancer 100: 2362-2366, 2004.

7. Jiang YW and Chen LA: microRNAs as tumor inhibitors, oncogenes, biomarkers for drug efficacy and outcome predictors in lung cancer (review). Mol Med Rep 5: 890-894, 2012.

8. Fang YX and Gao WQ: Roles of microRNAs during prostatic tumorigenesis and tumor progression. Oncogene 2013.

9. Hamamoto J, Soejima K, Yoda S, et al: Identification of microRNAs differentially expressed between lung squamous cell carcinoma and lung adenocarcinoma. Mol Med Rep 8: 456-462, 2013.
10. Nazarov PV, Reinsbach SE, Muller A, et al: Interplay of microRNAs, transcription factors and target genes: linking dynamic expression changes to function. Nucleic Acids Res 41: 2817-2831, 2013.

11. Marcucci G, Maharry KS, Metzeler KH, et al: Clinical role of microRNAs in cytogenetically normal acute myeloid leukemia: miR-155 upregulation independently identifies high-risk patients. J Clin Oncol 31: 2086-2093, 2013.

12. Yang M, Shen H, Qiu C, et al: High expression of miR-21 and miR-155 predicts recurrence and unfavourable survival in non-small cell lung cancer. Eur J Cancer 49: 604-615, 2013.

13. Huang X, Taeb S, Jahangiri S, et al: MiRNA-95 mediates radioresistance in tumors by targeting the sphingolipid phosphatase SGPP1. Cancer Res 2013.

14. Huang $X$ and Jia Z: Construction of HCC-targeting artificial miRNAs using natural miRNA precursors. Exp Ther Med 6: 209-215, 2013.

15. Shi XB, Xue L, Ma AH, et al: MiR-125b promotes growth of prostate cancer xenograft tumor through targeting pro-apoptotic genes. Prostate 71: 538-549, 2011.

16. Zhang CM, Zhao J and Deng HY: MiR-155 promotes proliferation of human breast cancer MCF-7 cells through targeting tumor protein 53 -induced nuclear protein 1. J Biomed Sci 20: 79, 2013.

17. Powell-Coffman JA and Coffman CR: Apoptosis: Lack of oxygen aids cell survival. Nature 465: 554-555, 2010.

18. Li T, Kon N, Jiang L, et al: Tumor suppression in the absence of p53-mediated cell-cycle arrest, apoptosis, and senescence. Cell 149: 1269-1283, 2012.

19. Petrocca F, Visone R, Onelli MR, et al: E2F1-regulated microRNAs impair TGFbeta-dependent cell-cycle arrest and apoptosis in gastric cancer. Cancer Cell 13: 272-286, 2008.

20. Higgs $G$ and Slack F: The multiple roles of microRNA-155 in Oncogenesis. J Clin Bioinforma 3: 17, 2013.

21. Streicher WW, Lopez MM and Makhatadze GI: Annexin I and annexin II N-terminal peptides binding to S100 protein family members: specificity and thermodynamic characterization. Biochemistry 48: 2788-2798, 2009.

22. Lokman NA, Ween MP, Oehler MK and Ricciardelli C: The role of annexin A2 in tumorigenesis and cancer progression. Cancer Microenviron 4: 199-208, 2011.

23. Paweletz CP, Ornstein DK, Roth MJ, et al: Loss of annexin 1 correlates with early onset of tumorigenesis in esophageal and prostate carcinoma. Cancer Res 60: 6293-6297, 2000.

24. Jin Y, Wang S, Chen W, et al: Annexin A7 suppresses lymph node metastasis of hepatocarcinoma cells in a mouse model. BMC Cancer 13: 522, 2013.

25. Srivastava M, Bubendorf L, Srikantan V, et al: ANX7, a candidate tumor suppressor gene for prostate cancer. Proc Natl Acad Sci USA 98: 4575-4580, 2001. 